\title{
Analgesic and anti-inflammatory effects in animal models of an ethanolic extract of Taheebo, the inner bark of Tabebuia avellanedae
}

\author{
MU HONG LEE ${ }^{1,2}$, HYUN MI CHOI ${ }^{1}$, DAE-HYUN HAHM ${ }^{3}$, ERK HER $^{4}$, \\ HYUNG-IN YANG ${ }^{5}$, MYUNG CHUL YOO ${ }^{1}$ and KYOUNG SOO KIM ${ }^{1}$
}

\begin{abstract}
${ }^{1}$ East-West Bone and Joint Research Institute, Kyung Hee University, Seoul 134-727; ${ }^{2}$ Korea Apiculture Agricultural Cooperative, Ansung 456-843; ${ }^{3}$ Acupuncture and Meridian Science Research Center, Kyung Hee University, Seoul 130-701; ${ }^{4}$ Department of Immunology, School of Medicine, Konkuk University, Chungju 380-701; ${ }^{5}$ Department of Internal Medicine, Kyung Hee University Hospital at Gangdong, Seoul 134-727, Republic of Korea
\end{abstract}

Received February 29, 2012; Accepted July 9, 2012

DOI: $10.3892 / \mathrm{mmr} .2012 .989$

\begin{abstract}
Taheebo, the purple inner bark of the Bignoniaceae tree Tabebuia avellanedae Lorentz ex Griseb, which is found in tropical rain forests in northeastern Brazil, has been used as a traditional medicine for various diseases for more than 1,500 years. In the current study, various animal models were used to demonstrate the analgesic and anti-inflammatory properties of its ethanolic extract, thereby investigating its potential as a therapeutic treatment for diseases with pain and inflammation. In the hot plate and writhing tests for the in vivo analgesic effect test of Taheebo, a $200 \mathrm{mg} / \mathrm{kg}$ dose of the extract induced a significant anti-nociceptive effect and increased the pain threshold by approximately $30 \%$ compared with the control. In vascular permeability and tetradecanoylphorbol acetate (TPA)-, arachidonic acid- and carrageenan-induced paw edema tests for anti-inflammatory effects, treatment with $200 \mathrm{mg} / \mathrm{kg}$ Taheebo led to significant anti-inflammatory effects and inhibited inflammation by $30-50 \%$ compared with the control. At $100 \mathrm{mg} / \mathrm{kg}$, the extract decreased the levels of pain and inflammation in all tested models, but the degree of inhibition was not statistically significant. The results suggest that the ethanolic extract of the inner bark of Tabebuia avellanedae has the potential to be developed as a therapeutic or supportive drug against diseases with accompanying pain and inflammation, including osteoarthritis.
\end{abstract}

\section{Introduction}

Among the elderly, osteoarthritis is the most common joint disease and a significant cause of physical illness (1). Symptoms

Correspondence to: Dr Kyoung Soo Kim, East-West Bone and Joint Research Institute, Kyung Hee University, 149 Sangil-dong, Gangdong-gu, Seoul 134-727, Republic of Korea

E-mail: labrea46@yahoo.co.kr

Key words: Tabebuia avellanedae Lorentz ex Griseb, Taheebo, anti-inflammatory effect, anti-nociceptive effect include joint pain, stiffness, limited movement, joint deformity and varying degrees of joint inflammation $(2,3)$. Current therapeutic strategies for osteoarthritis focus on alleviating symptoms, particularly pain and inflammation. Non-steroidal anti-inflammatory drugs (NSAIDs) and corticosteroids are the mainstream treatments for osteoarthritis (4). Although NSAIDs are recommended as an initial drug therapy to reduce joint inflammation and pain, their chronic use is limited by gastrointestinal-related toxicities, including nausea, dyspepsia, upper gastrointestinal bleeding and ulcer perforation (5). To minimize these toxicities, a new generation of NSAIDs, the cyclooxygenase (COX)-2 selective inhibitors (celecoxib, rofecoxib and valdecoxib), have been developed in an attempt to improve gastrointestinal tolerance. However, reported cardiovascular risks, including myocardial infarction and stroke, have led to the removal of rofecoxib from the market (6-8). Although other COX-2 inhibitors provide effective symptomatic relief, their substantial toxicities limit long-term use. Additionally, this therapeutic approach is not curative, but relieves clinical signs and symptoms of the disease; thus, a more effective and safe drug is necessary for the curative treatment of osteoarthritis.

Joins $^{\mathrm{TM}}$, a herbal drug combining the extracts of Clematis mandshurica, Trichosanthes kirilowii and Prunella vulgaris, is commonly used for the curative treatment of osteoarthritis in Korea. Clinical studies have demonstrated that Joins relieves joint pain and improves functionality in osteoarthritis patients. Its efficacy may be attributed to cartilage protection and anti-inflammation (9); however, its lack of an immediate analgesic effect is a major drawback. The screening of herbs and natural products for a more efficient compound may lead to the development of a superior therapeutic drug, particularly one with more immediate analgesic effects.

Tabebuia avellanedae Lorentz ex Griseb, a Bignoniaceae, is a tree found in tropical rain forests in northeastern Brazil. Taheebo, a product obtained from the purple bark of the tree, has been traditionally used for over 1,500 years in South America to treat a variety of diseases (10). Its various fractions have been previously reported to exhibit anti-inflammatory, anti-bacterial, anti-fungal, diuretic, anti-coagulant and laxative properties in addition to an anticancer effect (11-14). In 
particular, Taheebo demonstrated anti-nociceptive and anti-edematogenic effects in formalin- or acetic acid-induced nociceptive experimental models in mice and in the rat paw edema model (15). Compared with the anti-inflammatory effect of Tabebuia avellanedae, the analgesic effects of Taheebo have not been extensively studied to date (16). For the development as a therapeutic drug against osteoarthritis, the anti-inflammatory and analgesic effects of Taheebo ethanolic extract require further evaluation with various nociceptive and inflammatory experimental animal models. The current study investigated the anti-inflammatory and anti-nociceptive effects of the ethanolic extract of Tabebuia avellanedae on various animal models.

\section{Materials and methods}

Chemicals. Arachidonic acid, 12-O-tetradecanoylphorbol-13acetate (TPA), acetic acid, carboxymethyl cellulose (CMC), Evans blue, diclofenac, indomethacine and carrageenan were purchased from Sigma-Aldrich (St. Louis, MO, USA).

Animals. Male 6- to 8-week-old imprinting control region (ICR) mice and Sprague Dawley (SD) rats were obtained from OrientBio Co., Ltd. (Sungnam, Korea). Male ICR mice were used in the following tests: TPA- or arachidonic acid-induced ear edema, hot plate, acetic acid-induced vascular permeability and acetic acid-induced writhing response. SD rats were used in carrageenan-induced paw edema tests. The animals were maintained in plastic cages at $21-24^{\circ} \mathrm{C}$ under a 12 -h light/dark cycle and were provided with free access to pellet food and water. The animals were adapted for at least 1 week prior to the start of the experiment. The subjects were habituated to the behavioral test chambers and handled with special care to minimize stress. All methods were approved by the Animal Care and Use Committee of Kyung Hee University and all procedures were conducted in accordance with the Guide for the Care and Use of Laboratory Animals, published by the Korean National Institute of Health.

Preparation of ethanolic extract of Taheebo. An ethanolic extract from Taheebo, the inner bark of Tabebuia avellanedae Lorentz ex Griseb, was prepared as previously reported (17). Briefly, the inner bark of the plant was purchased from GIPI Korea Co. Ltd. (Seoul, Korea), who authenticated the product as Taheebo. Dried inner bark $(1 \mathrm{~kg})$ was extracted three times with $70 \%$ ethanol (12 1) at room temperature for 3 days. The combined extract was filtered and then concentrated under reduced pressure at $40^{\circ} \mathrm{C}$ with an end result of $\sim 13 \%$ yield (based on the weight of the dried inner bark). The extract was suspended in $0.5 \%$ sodium CMC (CMC-Na) solution immediately prior to the start of the experiments. The extract was administered orally to the animals at doses of 100, 200 or $400 \mathrm{mg} / \mathrm{kg}$. Control experiments were performed with $0.5 \%$ CMC-Na solution. A combination of diclofenac $(25 \mathrm{mg} / \mathrm{kg})$ and indomethacin $(1 \mathrm{mg} / \mathrm{kg})$ was used as the positive control.

Hot plate test. The hot plate test was conducted at a fixed temperature of $55 \pm 1^{\circ} \mathrm{C}$ according to a previously reported method (18). Animals were placed into a $50 \mathrm{~cm}$-diameter glass beaker on the heated surface and the time between animal placement and shaking or licking of the paws or jumping was recorded as the index of response latency. Mice exhibiting latency time of 10-30 sec were selected to receive vehicle (0.5\% CMC in distilled water), Taheebo extract (100, 200 or $400 \mathrm{mg} / \mathrm{kg})$ or diclofenac $(25 \mathrm{mg} / \mathrm{kg}) ; 1 \mathrm{~h}$ later, the latency time was re-determined.

Acetic acid-induced writhing response. The anti-nociceptive activities of the Taheebo extract were assessed by measuring the response to an intraperitoneal injection of acetic acid solution, which causes contraction of the abdominal muscles and stretching of the hind limbs (19). Each experimental group was administered orally with the vehicle $(0.5 \% \mathrm{CMC}$ in distilled water), Taheebo extract $(100,200$ or $400 \mathrm{mg} / \mathrm{kg})$, or diclofenac (25 mg/kg). After $1 \mathrm{~h}, 1 \%$ acetic acid was administered at a dose of $10 \mathrm{ml} / \mathrm{kg}$ body weight; measurement of the number of writhes began $10 \mathrm{~min}$ later and continued for $20 \mathrm{~min}$.

Acetic acid-induced vascular permeability. The acetic acid-induced vascular permeability test was carried out with a modification of a previously described method (20). Evans blue $(10 \mathrm{ml} / \mathrm{kg}$ of $2 \%$ solution) was injected intravenously into each mouse tail 30 min following oral administration of vehicle $(0.5 \% \mathrm{CMC}$ in distilled water), Taheebo extract (100, 200 or $400 \mathrm{mg} / \mathrm{kg}$ ) or diclofenac $(25 \mathrm{mg} / \mathrm{kg})$. After $20 \mathrm{~min}$, $10 \mathrm{ml} / \mathrm{kg}$ of $1 \%$ acetic acid in saline was injected intraperitoneally and the mice were sacrificed by cervical dislocation 20 min later. Saline $(5 \mathrm{ml})$ was injected into the abdominal cavity, the washings were collected into test tubes and subsequently centrifuged at 2,000 rpm for $10 \mathrm{~min}$. The absorbance of the supernatant was read at $650 \mathrm{~nm}$ with a SpectraMax 190 spectrophotometer (Molecular Devices, Sunnyvale, CA, USA) and the amount of Evans blue leakage in the abdominal cavity (the vascular permeability) was determined from the absorbance measurement of the supernatant.

TPA-induced ear edema formation in mice. Skin inflammation was induced in the right ear of each mouse by topical application of TPA as previously described (21). Each experimental group orally received the vehicle $(0.5 \% \mathrm{CMC}$ in distilled water), Taheebo extract $(100,200$ or $400 \mathrm{mg} / \mathrm{kg})$ or indomethacin $(1 \mathrm{mg} / \mathrm{kg})$. After $1 \mathrm{~h}$, TPA solution $(1.0 \mu \mathrm{g}$ dissolved in $20 \mu \mathrm{l}$ acetone) was applied to the inner and outer surfaces of the ears and $4 \mathrm{~h}$ later the mice were sacrificed by cervical dislocation. A mouse ear punch was obtained with a $5-\mathrm{mm}$ dermal biopsy punch and then weighed. The thickness of the punch was measured with calipers (Mitutoyo Corporation, Kawasaki, Japan). The degree of ear swelling was expressed as the increase in ear thickness (mm).

Arachidonic acid-induced mouse ear edema assay. The vehicle (0.5\% CMC in distilled water), Taheebo extract (100, 200 or $400 \mathrm{mg} / \mathrm{kg}$ ) or indomethacin (1 mg/kg) were orally administered $1 \mathrm{~h}$ prior to the topical application of $2 \%$ arachidonic acid dissolved in acetone ( $20 \mu \mathrm{l} / \mathrm{ear})$ to the right ear of the mice (22). After $4 \mathrm{~h}$, the mice were sacrificed by cervical dislocation. A mouse ear punch was obtained with a 5-mm dermal biopsy punch and then weighed. The thickness of the punch was measured with calipers and the degree of ear swelling was expressed as the increase in ear thickness ( $\mathrm{mm})$. 
Carrageenan-induced paw edema. The anti-inflammatory activity of Taheebo was determined by the carrageenan-induced edema test. Taheebo extract $(100,200$ or $400 \mathrm{mg} / \mathrm{kg})$, diclofenac $(25 \mathrm{mg} / \mathrm{kg})$ or the vehicle $(0.5 \% \mathrm{CMC}$ in distilled water) was administered orally $1 \mathrm{~h}$ prior to the injection of $100 \mu \mathrm{l}$ of $1 \%$ carrageenan in saline into the plantar side of the left hind paws of the rats. Paw volume was measured prior to the carrageenan injection and 1,2, 3 and $4 \mathrm{~h}$ following the administration of the edematogenic agent using a plethysmometer (Ugo Basile, Comerio, Italy). The degree of swelling was determined by the ratio $a / b$, where $a$ and $b$ are the volumes of the left hind paws following and prior to the carrageenan treatment, respectively. The increase in paw volume (\%) was calculated as follows: [(a-b)/b] x 100 .

Statistical analysis. Data were presented as the mean \pm standard error of the mean (SEM). Comparisons between the experimental and control groups were performed by one-way analysis of variance (ANOVA) followed by Dunnett's post hoc test. $\mathrm{P}<0.05$ was considered to indicate a statistically significant result. The program used for the statistical analysis was GraphPad Prism software 5 (San Diego, CA, USA).

\section{Results}

Analgesic effect of the ethanolic Taheebo extract in animal models. Oral administration of the Taheebo extract significantly increased the pain threshold of the mice compared with control treatment, as assessed by the hot plate test (Fig. 1). Notably, treatment with Taheebo led to a significant analgesic effect even at $200 \mathrm{mg} / \mathrm{kg}$ ( $\sim 30 \%$ greater than control), an effect that was similar to treatment with diclofenac $(25 \mathrm{mg} / \mathrm{kg})$. The acetic acid-induced writhing response also revealed the analgesic effect of Taheebo. The cumulative amount of abdominal stretching was associated with the level of acetic acid-induced pain. Thus, Taheebo treatment (100-400 mg/kg) significantly inhibited the number of writhes in comparison to control treatment (Fig. 2). The inhibition of writhes was $\sim 30$ and $40 \%$ higher than the control at Taheebo doses of 100 and $400 \mathrm{mg} / \mathrm{kg}$, respectively, although this inhibition did not achieve statistical significance at $100 \mathrm{mg} / \mathrm{kg}$.

Anti-inflammatory effect of ethanolic Taheebo extract in animal models. The anti-inflammatory effects of Taheebo extract were assessed through the use of various animal models, including acetic acid-induced vascular permeability, TPA-induced ear edema, arachidonic acid-induced mouse ear edema and carrageenan-induced paw edema. First, in the acetic acid-induced vascular permeability test, the oral administration of Taheebo extract at 100 and $400 \mathrm{mg} / \mathrm{kg}$ resulted in the significant inhibition of dye leakage by 30 and $35 \%$, respectively (Fig. 3). The inhibition degree was similar to $100-400 \mathrm{mg} / \mathrm{kg}$, although only the inhibition at $400 \mathrm{mg} / \mathrm{kg}$ achieved statistical significance. In the TPA-induced ear edema test, the Taheebo extract led to an inhibition of ear weight and thickness in a dose-dependent manner (Fig. 4). In the arachidonic acid-induced mouse ear edema model, Taheebo extract also exhibited a dose-dependent anti-inflammatory effect (Fig. 5). In the two models, indomethacin $(1 \mathrm{mg} / \mathrm{kg})$ inhibited ear inflammation by $50 \%$ compared with the control, while the Taheebo extract at $100 \mathrm{mg} / \mathrm{kg}$

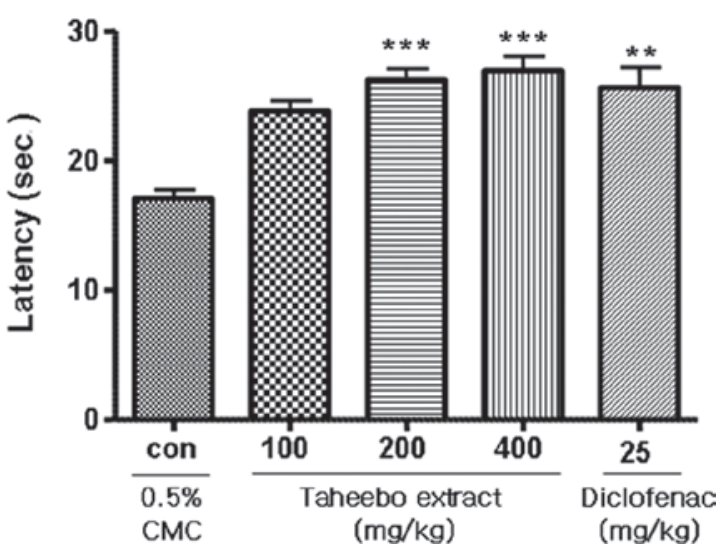

Figure 1. Analgesic effects of Taheebo extract on thermally-induced pain in mice. The hot plate response latency was measured $1 \mathrm{~h}$ after oral administration of Taheebo extract $(100,200$ or $400 \mathrm{mg} / \mathrm{kg})$, the positive control diclofenac $(25 \mathrm{mg} / \mathrm{kg})$ or the negative control $(0.5 \% \mathrm{CMC}$ in distilled water). Values are expressed as the means \pm SEM $(n=9) .{ }^{* *} \mathrm{P}<0.01$ and ${ }^{* * *} \mathrm{P}<0.001$ indicate statistically significant differences compared to the vehicle control group. CMC, carboxymethyl cellulose.

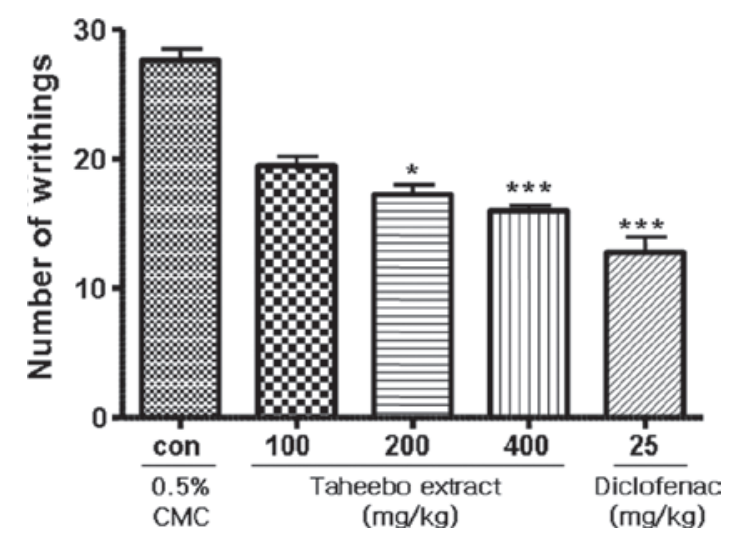

Figure 2. Analgesic effects of the Taheebo extract on acetic acid-induced pain in mice. Acetic acid-induced writhing number was measured $1 \mathrm{~h}$ after oral administration of the extract or controls, as described in Fig. 1. Acetic acid $(1 \%)$ at a dose of $10 \mathrm{ml} / \mathrm{kg}$ was injected and $10 \mathrm{~min}$ later, the number of writhes was recorded during the subsequent $20-\mathrm{min}$ period. The values are expressed as the means \pm SEM $(n=9)$. ${ }^{*} \mathrm{P}<0.05,{ }^{* *} \mathrm{P}<0.01$ and ${ }^{* * *} \mathrm{P}<0.001$ indicate statistically significant differences compared to the vehicle control group. CMC, carboxymethyl cellulose.

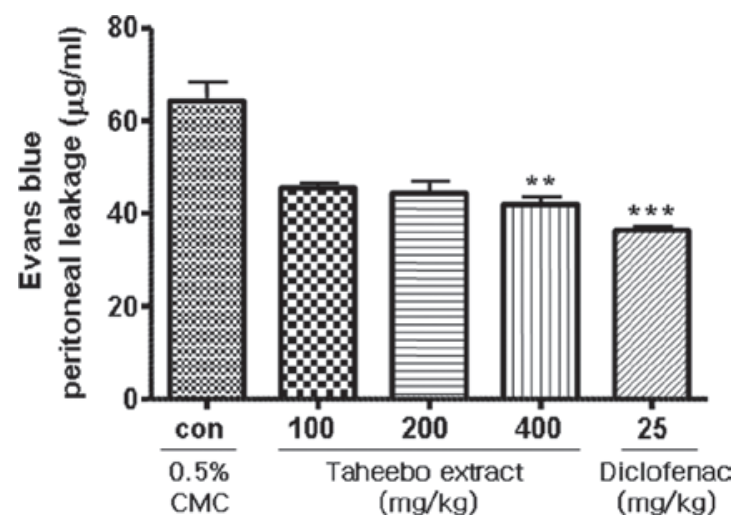

Figure 3. Anti-inflammatory effect of Taheebo extract on acetic acid-induced vascular permeability. The amount of Evans blue leakage in the abdominal cavity was measured as an indicator of inflammation degree. The values are expressed as the means $\pm \operatorname{SEM}(\mathrm{n}=9) .{ }^{* *} \mathrm{P}<0.01$ and ${ }^{* * *} \mathrm{P}<0.001$ indicate statistically significant differences compared to the vehicle control group. CMC, carboxymethyl cellulose. 
Table I. Anti-inflammatory effect of Taheebo extract on carrageenan-induced paw edema in rats.

Percentage increase of paw edema (mean \pm SEM)

\begin{tabular}{lcccc}
\cline { 2 - 5 } Dose $(\mathrm{mg} / \mathrm{kg})$ & $1 \mathrm{~h}$ & $2 \mathrm{~h}$ & $3 \mathrm{~h}$ & $4 \mathrm{~h}$ \\
\hline CON & $29.36 \pm 2.66$ & $59.57 \pm 3.10$ & $76.28 \pm 2.24$ & $76.14 \pm 2.52$ \\
Taheebo (100) & $29.47 \pm 1.89$ & $56.24 \pm 2.31$ & $62.23 \pm 3.95$ & $64.04 \pm 2.77$ \\
Taheebo (200) & $30.73 \pm 2.12$ & $50.58 \pm 2.60^{\mathrm{a}}$ & $57.86 \pm 2.43^{\mathrm{b}}$ & $60.78 \pm 2.22^{\mathrm{b}}$ \\
Taheebo (400) & $30.60 \pm 2.11$ & $47.10 \pm 2.27^{\mathrm{b}}$ & $51.32 \pm 2.72^{\mathrm{b}}$ & $52.19 \pm 2.30^{\mathrm{b}}$ \\
Diclofenac (25) & $27.83 \pm 2.82$ & $37.98 \pm 2.37^{\mathrm{b}}$ & $41.18 \pm 2.93^{\mathrm{b}}$ & $35.23 \pm 2.69^{\mathrm{b}}$
\end{tabular}

Values are expressed as the means $\pm \operatorname{SEM}(\mathrm{n}=9) .{ }^{\mathrm{a}} \mathrm{P}<0.05,{ }^{\mathrm{b}} \mathrm{P}<0.01$ and ${ }^{\mathrm{c}} \mathrm{P}<0.001$ indicate statistically significant differences from the vehicle control group. CON, $0.5 \%$ CMC-Na solution.

A

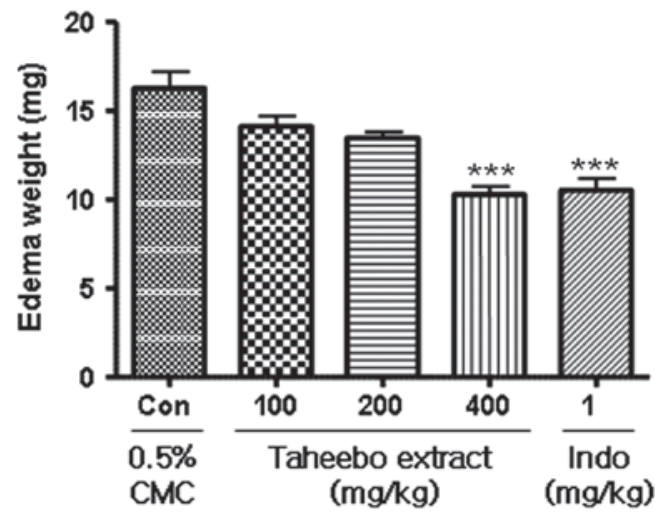

B

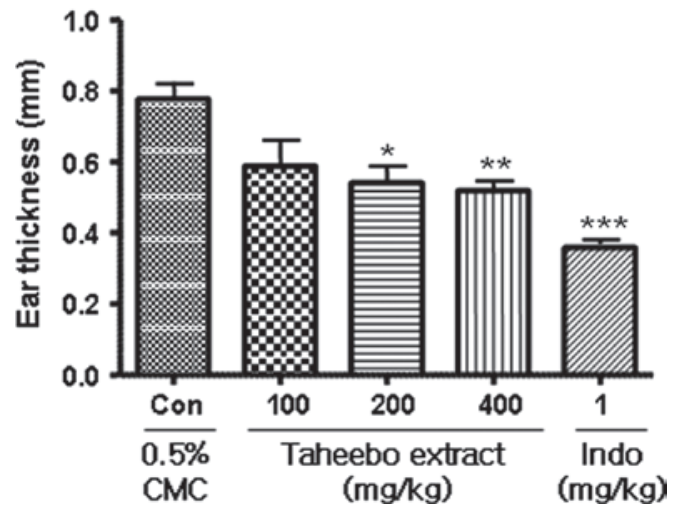

Figure 4. Anti-inflammatory effect of Taheebo extract on TPA-induced ear skin inflammation in mice. The (A) weight and (B) thickness of ear punches were measured as an indicator of the inflammation degree. The values are expressed as the means \pm SEM $(n=9) .{ }^{* *} \mathrm{P}<0.01$ and ${ }^{* * * *} \mathrm{P}<0.001$ indicate statistically significant differences compared to the vehicle control group. CMC, carboxymethyl cellulose; indo, indomethacine.

inhibited ear inflammation by $>25 \%$ compared with the control. In the carrageenan-induced hind paw edema test, $200 \mathrm{mg} / \mathrm{kg}$ Taheebo extract significantly inhibited $\sim 30 \%$ of paw edema $3 \mathrm{~h}$ following carrageenan injection, although $100 \mathrm{mg} / \mathrm{kg}$ Taheebo extract was unable to significantly decrease edema volume compared with the control at all time points (Table I).

\section{Discussion}

Numerous studies have elucidated the pharmacological activities of the Taheebo extract, the inner bark of Tabebuia
A
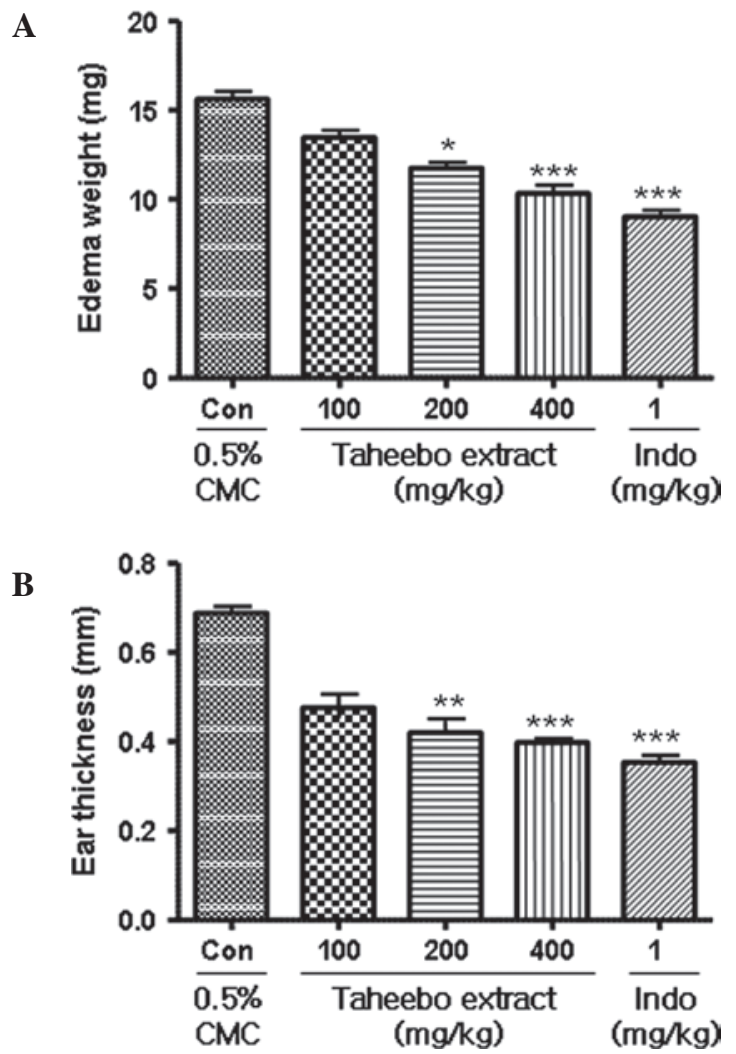

Figure 5. Anti-inflammatory effect of Taheebo extract on arachidonic acid-induced ear skin inflammation in mice. The (A) weight and (B) thickness of ear punches were measured to assess the degree of inflammation following experimental or control treatment. The values are expressed as the means \pm SEM $(n=9) .{ }^{*} \mathrm{P}<0.05,{ }^{* * *} \mathrm{P}<0.01$ and ${ }^{* * *} \mathrm{P}<0.001$ indicate statistically significant differences compared to the vehicle control group. CMC, carboxymethyl cellulose; indo, indomethacine.

avellanedae (11-14). However, the few demonstrations of the in vivo anti-nociceptive effects were performed in limited animal models (15). Those studies focused on the aqueous and methanolic extracts of Tabebuia avellanedae; to the best of our knowledge, the ethanolic extracts have not undergone much study. This investigation has demonstrated that the ethanolic extract of Taheebo significantly attenuated acetic acid-induced writhing (Fig. 2) and the nociception produced by hot-plate thermal stimulation (Fig. 1). Treatment with the extract also decreased the inflammation induced by acetic 
acid (Fig. 3), TPA (Fig. 4), arachidonic acid (Fig. 5) and carrageenan (Table I) in animal models.

For nociception induction in animals, acetic acid causes inflammatory pain by inducing capillary permeability (23), while hot-plate-induced pain indicates narcotic involvement (24). Although formalin is known to induce neurogenic and inflammatory pain (25), the formalin test was not performed in this study. However, the demonstration that Taheboo aqueous extract also exerts an analgesic effect on formalin-induced pain (15) suggests that the Taheebo extract has an inhibitory effect on three types of pain induction. These analgesic effects may be partly related to Taheebo's anti-inflammatory neurogenic and narcotic properties. Oral administration of $200-400 \mathrm{mg} / \mathrm{kg}$ of the ethanolic Taheebo extract inhibited pain with statistical significance in the two animal models and a dose of $100 \mathrm{mg} / \mathrm{kg}$ was extremely effective in the majority of tested animal models. This inhibitory concentration is similar to that of the aqueous extract of Tabebuia avellanedae (15). Notably, the inhibitory effect of this aqueous extract at $200 \mathrm{mg} / \mathrm{kg}$ was not detected following the $400 \mathrm{mg} / \mathrm{kg}$ dose, as the aqueous extract may contain compounds that undercut its own inhibitory action. By contrast, in this study the ethanolic extract caused significant anti-nociceptive effects at doses of $200-400 \mathrm{mg} / \mathrm{kg}$ in various animal models, suggesting that the ethanolic extracts contain constituents to relieve pain that differ from the aqueous extracts.

The molecular mechanism by which the extract attenuated the pain level in the animal models has not been defined. Collier et al postulated that acetic acid acts indirectly by inducing the release of endogenous mediators that stimulate nociceptive neurons sensitive to NSAIDs and narcotics (26). It has also been proposed that acetic acid induces pain by increasing the amount of $\mathrm{PGE}_{2}$ and $\mathrm{PGF}_{2 \alpha}$ at the peritoneal receptors $(27,28)$. On the basis of these studies, the Taheebo extract is thought to regulate the production of $\mathrm{PGE}_{2}$ and $\mathrm{PGF}_{2 \alpha}$ in acetic acidinduced animal models of pain. By contrast, as the hot plate test is commonly used for assays of narcotic analgesics, our observations suggest that the extract exerts a central anti-nociceptive effect that may be associated with a reduction in $\mathrm{Ca}^{2+}$ influx at the axon termini of the afferent nerves. This reduction may induce a decrease in adenylate cyclase activity, thereby decreasing the levels of cyclic AMP and $\mathrm{K}^{+}$efflux and leading to nerve hyperpolarization and an apparent anti-nociceptive effect (29).

Carrageenan, arachidonic acid, TPA and acetic acid were used to induce inflammation in animal models, since edema induced by phlogistic agents is a widely accepted model for inflammation. For example, carrageenan-induced paw edema is a classical model of acute inflammation involving various chemical mediators, including histamine, serotonin, bradykinin and prostgladins (30), in which the involvement of the COX products of arachidonic acid metabolism and the production of reactive oxygen species are well-established (31). Oral administration of the Taheebo extract inhibited the inflammation in these animal models and thus the extract may be presumed to be involved in the downregulation of the production of various chemical mediators of inflammation.

The ethanolic extract of Taheebo exhibited anti-nociceptive and anti-inflammatory effects in animal models, emphasizing its potential for development as a novel therapeutic drug against osteoarthritis. However, studies using other pain and inflammation animal models are required, particularly since the animal models used in this investigation were short-term. The effects of lower doses of Taheebo should be demonstrated in long-term animal models to support its development as a therapeutic drug.

The following major active compounds have been identified in Tabebuia avellandae: flavonoids, cyclopentene dialdehydes, benzoic acid and benzaldehyde derivatives, quinones, furanonaphthoquinones, naphthoquinones and anthraquinones (32). Of the 18 most relevant quinines, lapachol and $\beta$-lapachone appear to have clinical importance, since they have been associated with anticancer pharmacological activity. Thus, HPLC analysis was conducted to determine the content of lapachol in the ethanol extract of Taheebo (data not shown). Lapachol was detected at 6.642 min retention time. The extract $(1 \mathrm{~g})$ contained approximately $0.03 \mathrm{mg}$ of lapachol, although we did not confirm the content of all the compounds. Toxicity was observed with no therapeutic response following oral administration of lapachol and phase I clinical trials of lapachol as an anticancer drug were closed in 1970. The anticancer chemotherapeutic $\beta$-lapachone succeeded as the research molecule of note from Tabebuia avellandae and the molecular mechanisms associated with its anticancer activity, including topoisomerase inhibition, have been well defined (32). Other compounds, including the furonaphthoquinones and anthraquinones, have been the focus of investigations for various therapeutic roles in several experimental systems. Other molecules considered to be significant in disease treatment should be studied to further understand the molecular mechanisms by which Taheebo extracts relieve pain and inflammation in animal models.

In conclusion, the Taheebo ethanolic extracts demonstrated anti-nociceptive and anti-inflammatory effects in various animal models. These anti-nociceptive effects and the extract constituents related to pain relief require clarification for the development of a safe drug that produces immediate analgesia without adverse effects.

\section{References}

1. Ameye LG and Chee WS: Osteoarthritis and nutrition. From nutraceuticals to functional foods: a systematic review of the scientific evidence. Arthritis Res Ther 8: R127, 2006.

2. Lark MW, Bayne EK, Flanagan J, et al: Aggrecan degradation in human cartilage. Evidence for both matrix metalloproteinase and aggrecanase activity in normal, osteoarthritic and rheumatoid joints. J Clin Invest 100: 93-106, 1997.

3. Poole AR: An introduction to the pathophysiology of osteoarthritis. Front Biosci 4: D662-D670, 1999.

4. Kidd BL: Osteoarthritis and joint pain. Pain 123: 6-9, 2006.

5. Wolfe F and Cathey MA: The assessment and prediction of functional disability in rheumatoid arthritis. J Rheumatol 18: 1298-1306, 1991.

6. Hippisley-Cox J and Coupland C: Risk of myocardial infarction in patients taking cyclo-oxygenase-2 inhibitors or conventional non-steroidal anti-inflammatory drugs: population based nested case-control analysis. BMJ 330: 1366, 2005.

7. Gottlieb S: COX 2 inhibitors may increase risk of heart attack. BMJ 323: 471, 2001

8. Lenzer J: FDA advisers warn: COX 2 inhibitors increase risk of heart attack and stroke. BMJ 330: 440, 2005.

9. Hartog A, Hougee S, Faber J, et al: The multicomponent phytopharmaceutical SKI306X inhibits in vitro cartilage degradation and the production of inflammatory mediators. Phytomedicine 15: 313-320, 2008.

10. Hashimoto G (ed): Illustrated Encyclopedia of Brazilian Medicinal Plants. Kamakura, Aboc-sha, 1996. 
11. Choi BT, Cheong J and Choi YH: Beta-Lapachone-induced apoptosis is associated with activation of caspase-3 and inactivation of NF- $\mathrm{KB}$ in human colon cancer HCT-116 cells. Anticancer Drugs 14: 845-850, 2003.

12. Machado TB, Pinto AV, Pinto MC, et al: In vitro activity of Brazilian medicinal plants, naturally occurring naphthoquinones and their analogues, against methicillin-resistant Staphylococcus aureus. Int J Antimicrob Agents 21: 279-284, 2003.

13. Awale S, Kawakami T, Tezuka Y, et al: Nitric oxide (NO) production inhibitory constituents of Tabebuia avellanedae from Brazil. Chem Pharm Bull (Tokyo) 53: 710-713, 2005.

14. Böhler T, Nolting J, Gurragchaa P, et al: Tabebuia avellanedae extracts inhibit IL-2-independent T-lymphocyte activation and proliferation. Transpl Immunol 18: 319-323, 2008.

15. de Miranda FG, Vilar JC, Alves IA, et al: Antinociceptive and antiedematogenic properties and acute toxicity of Tabebuia avellanedae Lor. ex Griseb. inner bark aqueous extract. BMC Pharmacol 1: 6, 2001.

16. Byeon SE, Chung JY, Lee YG, et al: In vitro and in vivo antiinflammatory effects of taheebo, a water extract from the inner bark of Tabebuia avellanedae. J Ethnopharmacol 119: 145-152, 2008.

17. Lee KH and Choi EM: Analgesic and anti-inflammatory effects of Ligularia fischeri leaves in experimental animals. J Ethnopharmacol 120: 103-107, 2008.

18. $\mathrm{Hu} \mathrm{X}$, Jin $\mathrm{H}, \mathrm{Xu} \mathrm{W}$, et al: Anti-inflammatory and analgesic effects of Daphne retusa Hemsl. J Ethnopharmacol 120: 118-122, 2008.

19. Fischer LG, Santos D, Serafin C, et al: Further antinociceptive properties of extracts and phenolic compounds from Plinia glomerata (Myrtaceae) leaves. Biol Pharm Bull 31: 235-239, 2008

20. Whittle BA: The use of changes in capillary permeability in mice to distinguish between narcotic and nonnarcotic analgesics. Br J Pharmacol Chemother 22: 246-253, 1964.

21. Song HY, Lee JA, Ju SM, et al: Topical transduction of superoxide dismutase mediated by HIV-1 Tat protein transduction domain ameliorates 12-O-tetradecanoylphorbol-13-acetate (TPA)-induced inflammation in mice. Biochem Pharmacol 75: 1348-1357, 2008
22. Koshihara Y, Fujimoto Y and Inoue H: A new 5-lipoxygenase selective inhibitor derived from Artocarpus communis strongly inhibits arachidonic acid-induced ear edema. Biochem Pharmacol 37: 2161-2165, 1988.

23. Amico-Roxas M, Caruso A, Trombadore S, et al: Gangliosides antinociceptive effects in rodents. Arch Int Pharmacodyn Ther 272: 103-117, 1984.

24. Besra SE, Sharma RM and Gomes A: Antiinflammatory effect of petroleum ether extract of leaves of Litchi chinensis Gaertn. (Sapindaceae). J Ethnopharmacol 54: 1-6, 1996.

25. Vaz ZR, Filho VC, Yunes RA and Calixto JB: Antinociceptive action of 2-(4-bromobenzoyl)-3-methyl-4,6-dimethoxy benzofuran, a novel xanthoxyline derivative on chemical and thermal models of nociception in mice. J Pharmacol Exp Ther 278: 304-312, 1996.

26. Collier HO, Dinneen LC, Johnson CA and Schneider C: The abdominal constriction response and its suppression by analgesic drugs in the mouse. Br J Pharmacol Chemother 32: 295-310, 1968.

27. Deraedt R, Jouquey S, Delevallée F and Flahaut M: Release of prostaglandins $\mathrm{E}$ and $\mathrm{F}$ in an algogenic reaction and its inhibition. Eur J Pharmacol 61: 17-24, 1980.

28. Bentley GA, Newton SH and Starr J: Studies on the antinociceptive action of alpha-agonist drugs and their interactions with opioid mechanisms. Br J Pharmacol 79: 125-134, 1983.

29. Yaksh TL: Spinal systems and pain processing: development of novel analgesic drugs with mechanistically defined models. Trends Pharmacol Sci 20: 329-337, 1999.

30. Vinegar R, Truax JF, Selph JL, et al: Pathway to carrageenan-induced inflammation in the hind limb of the rat. Fed Proc 46: 118-126, 1987.

31. Smith MJ, Ford-Hutchinson AW, Elliott PN and Bolam JP: Prostaglandins and the anti-inflammatory activity of a human plasma fraction in carrageenan-induced paw oedema in the rat. J Pharm Pharmacol 26: 692-698, 1974.

32. Gomez Castellanos JR, Prieto JM and Heinrich M: Red Lapacho (Tabebuia impetiginosa) - a global ethnopharmacological commodity? J Ethnopharmacol 121: 1-13, 2009. 